International Journal of Applied Mathematics

Volume 31 No. $5 \quad 2018,569-584$

ISSN: 1311-1728 (printed version); ISSN: 1314-8060 (on-line version)

doi: http://dx.doi.org/10.12732/ijam.v31i5.3

\title{
EFFECT OF AN INFINITE SURFACE BREAKING, INCLINED, DIP-SLIP FAULT IN VISCOELASTIC HALF SPACE UNDER TECTONIC FORCES ON DISPLACEMENT, STRESS AND STRAIN
}

\author{
Debabrata Mondal ${ }^{1}$, Seema Sarkar (Mondal) ${ }^{1}$, Sanjay Sen ${ }^{2}$ \\ ${ }^{1}$ Department of Mathematics \\ National Institute of Technology \\ Durgapur - 720613, West Bengal, INDIA \\ ${ }^{2}$ Department of Applied Mathematics \\ University of Calcutta \\ Kolkata - 700009, West Bengal, INDIA
}

\begin{abstract}
A long, locked, dip-slip fault is considered situated in a half space of linear viscoelastic solid having the properties of both Maxwell and Kelvin (Voigt) type materials. Tectonic forces due to mantle convection and other associated phenomena are acting on the system. The magnitude of the tectonic forces has been assumed to be slowly increasing with time. The movement is assumed to be slipping in nature. Analytical expressions for the displacement, stresses and strains are obtained at any field point in an isotropic, homogeneous, viscoelastic half-space using integral transformation, modified Green's function technique and correspondence principle. A detailed study of these expressions may give some ideas about the nature of stress accumulation in the system.
\end{abstract}

AMS Subject Classification: 74D05, 86A17, 44A05

Key Words: dip-slip fault, isotropic medium, standard linear solid, Green's function technique, correspondence principle

\section{Introduction and Literature Review}

It is observational fact that while some faults are strike-slip in nature, there are faults (e.g. Sierra Nevada/Owen's valley; Basin and Range faults, Rocky

Received: April 13, 2018

(C) 2018 Academic Publications

${ }^{\S}$ Correspondence author 
Mountains, Himalayas; Atlantic fault of central Greece-steeply dipping faults with dip 60 degree, 80 degree), where the surface level changes during the motion i.e. the faults are dip-slip in nature. It is therefore necessary to understand the mechanism of plate motion in the dip direction with a displacement dislocation and the nature of stress-strain accumulation/release in spatial and temporal co-ordinate to predict the future event in space and time. A pioneering work involving static ground deformation in elastic media was initiated by [22], [23], [5], [12], [13]. In [18] the authors did a wonderful work in analyzing the displacement, stress and strain for dip-slip movement of the fault. Later some theoretical models in this direction have been formulated by a number of authors like [2], [6], [9], [10], [16], [17], [20], [24]. In the book [19] various aspects of fault movements have been discussed. [14], [15] have discussed stress accumulation near infinite fault in lithosphere-asthenosphere system. The works [7], [8], [21] have discussed about long dip-slip fault in the viscoelastic medium of Maxwell type material. In the earlier works, in most of the cases elastic or viscoelastic half space or layered medium were considered to represent the lithosphere-asthenosphere system. Observations in seismically active regions suggest that linear viscoelastic material of Maxwell and/ or Kelvin type may be a suitable representation of the system. In many cases tectonic forces are taken to be constant. In view of these, in this paper we consider an infinite sudden dip-slip movement situated in a linear viscoelastic solid combining both the properties of Maxwell and Kelvin type material. The system is under the action of tectonic forces generated due to mantle convection or similar other processes and displacements, stresses and strains are analyzed.

\section{Formulation}

We consider a long, dip-slip fault $F$, with width $D$ situated in a viscoelastic half space of linear Maxwell and Kelvin (Voigt) mixed type material. A Cartesian coordinate system is used with a suitable point $O$ as origin on the the fault, the strike of the fault is taken along $y_{1}$ axis, $y_{3}$ axis pointing downwards so that the free surface is given by $y_{3}=0$ and $y_{2}$ axis is perpendicular to $y_{1} y_{3}$ plane. We choose another coordinate system with $y_{1}^{\prime}, y_{2}^{\prime}, y_{3}^{\prime}$ axes as shown in Figure 1 so that the fault can be given by $F:\left(y_{2}^{\prime}=0,0 \leq y_{3}^{\prime} \leq D\right)$.

Let $\theta$ be the inclination of the fault $F$ with the free surface. We consider the section of the model by the plane $y_{1}=0$. The displacement, stress and strain components separate out into two independent groups. One group containing displacement $\mathrm{u}$, stress component $\left(\tau_{12}, \tau_{13}\right)$ and strain components $\left(E_{12}, E_{13}\right)$ 


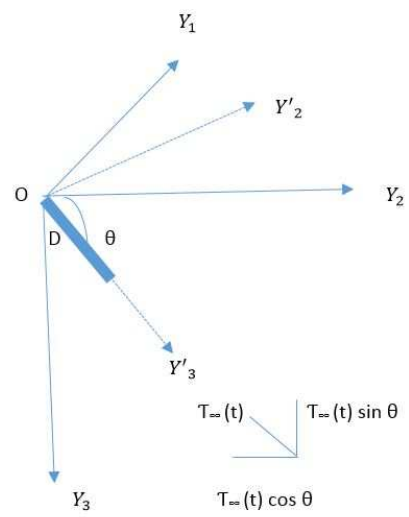

Figure 1: Coordinate system describing the location of the fault.

is associated with the strike slip movement while the other group containing displacement component $(v, w)$, stress components $\left(\tau_{22}, \tau_{23}, \tau_{33}\right)$ and strain components $\left(E_{22}, E_{23}, E_{33}\right)$ is associated with dip-slip movement. We consider the dip-slip movement across the fault $F$. Let $v, w$ be the displacement components along the $y_{2}, y_{3}$ axes, and $\tau_{22}, \tau_{23}, \tau_{33}$ are the stress components and $E_{22}, \quad E_{23}, \quad E_{33}$ are the strain components respectively. We take $t=0$ as an instant when the medium is in aseismic state.

\subsection{Constitutive Equation}

The stress-strain relationship for standard linear solid (SLS) of visco-elastic material can be represented by the following equation:

$$
\begin{aligned}
& \tau_{22}+\frac{\eta}{\mu} \frac{\partial}{\partial t}\left(\tau_{22}\right)=\mu \frac{\partial v}{\partial y_{2}}+2 \eta \frac{\partial}{\partial t}\left(\frac{\partial v}{\partial y_{2}}\right) \\
& \tau_{23}+\frac{\eta}{\mu} \frac{\partial}{\partial t}\left(\tau_{23}\right)=\frac{\mu}{2}\left(\frac{\partial v}{\partial y_{3}}+\frac{\partial w}{\partial y_{2}}\right)+2 \eta \frac{\partial}{\partial t} \frac{1}{2}\left(\frac{\partial v}{\partial y_{3}}+\frac{\partial w}{\partial y_{2}}\right) \\
& \text { From above, } \tau_{23} \text { can be written as } \\
& \tau_{23}+\frac{\eta}{\mu} \frac{\partial}{\partial t}\left(\tau_{23}\right)=\mu \frac{\partial v}{\partial y_{3}}+2 \eta \frac{\partial}{\partial t}\left(\frac{\partial v}{\partial y_{3}}\right) \\
& \tau_{23}+\frac{\eta}{\mu} \frac{\partial}{\partial t}\left(\tau_{23}\right)=\mu \frac{\partial w}{\partial y_{2}}+2 \eta \frac{\partial}{\partial t}\left(\frac{\partial w}{\partial y_{2}}\right) \\
& \tau_{33}+\frac{\eta}{\mu} \frac{\partial}{\partial t}\left(\tau_{33}\right)=\mu \frac{\partial w}{\partial y_{3}}+2 \eta \frac{\partial}{\partial t}\left(\frac{\partial w}{\partial y_{3}}\right)
\end{aligned}
$$


where $\eta$ is the effective viscosity and $\mu$ is the effective rigidity of the material.

\subsection{Stress Equation of Motion}

The stress satisfy the following equations (assuming quasi-static deformation for which the inertial terms are neglected) and the body forces do not change during our consideration:

$$
\left.\begin{array}{c}
\frac{\partial}{\partial y_{2}}\left(\tau_{22}\right)+\frac{\partial}{\partial y_{3}}\left(\tau_{23}\right)=0 \\
\frac{\partial}{\partial y_{2}}\left(\tau_{32}\right)+\frac{\partial}{\partial y_{3}}\left(\tau_{33}\right)=0
\end{array}\right\}
$$

where $\left(-\infty<y_{2}<\infty, \quad y_{3} \geq 0, \quad t \geq 0\right)$.

\subsection{Initial and Boundary Condition}

Let $(v)_{0},(w)_{0},\left(\tau_{22}\right)_{0},\left(\tau_{23}\right)_{0},\left(\tau_{33}\right)_{0},\left(E_{22}\right)_{0},\left(E_{23}\right)_{0},\left(E_{33}\right)_{0}$ be the initial values of $v, w, \tau_{22}, \tau_{23}, \tau_{33}, E_{22}, E_{23}, E_{33}$ respectively at time $t=0$. Tectonic forces far away from the fault due to mantle convection in the lithosphere-asthenosphere system cause the fault to slip leading to an earthquake. We represent this tectonic forces by $\tau_{\infty}(t)=\tau_{\infty}(0)(1+K t)$, where $K$ is any positive number so that the tectonic forces increase linearly with time. The relevant boundary conditions become

$$
\tau_{22}\left(y_{2}, y_{3}, t\right)=\tau_{\infty}(0)(1+K t) \cos \theta, K>0
$$

as $\left|y_{2}\right| \rightarrow \infty, y_{3} \geq 0, t \geq 0$. On the free surface $y_{3}=0$,

$$
\tau_{23}\left(y_{2}, y_{3}, t\right)=0, \quad \tau_{33}\left(y_{2}, y_{3}, t\right)=0 \quad\left(-\infty<y_{2}<\infty, \quad t \geq 0\right) .
$$

Also as $y_{3} \rightarrow \infty$

$$
\tau_{23}\left(y_{2}, y_{3}, t\right)=0, \quad \tau_{33}\left(y_{2}, y_{3}, t\right)=\tau_{\infty}(0)(1+K t) \sin \theta, \quad K>0
$$

$\left(-\infty<y_{2}<\infty, \quad t \geq 0\right)$, where $\tau_{\infty}(0)$ is the value of $\tau_{\infty}(t)$ at $t=0$. The initial conditions satisfy all the above boundary conditions. 


\section{Solution}

Now differentiating partially 1 st equation of (1) with respect to $y_{2}$ and 3 rd equation of (1) with respect to $y_{3}$, adding them and using 1st equation of (2), we arrive at 1 st equation of (6). Similar other equation is given in 2 nd equation of (6). Thus,

$$
\left.\begin{array}{c}
\nabla^{2} V\left(y_{2}, y_{3}, t\right)=0, \text { where } V=v-(v)_{0} e^{-} \frac{\mu t}{2 \eta} \\
\nabla^{2} W\left(y_{2}, y_{3}, t\right)=0, \text { where } W=w-(w)_{0} e^{-\frac{\mu t}{2 \eta}}
\end{array}\right\} .
$$

\subsection{Solution Before any Fault Movement}

The boundary value problem given by equations (1) to (6) can be solved by taking Laplace transformation with respect to time $t$ of all the constitutive equations and the boundary conditions. On taking Laplace inverse transformation the solutions for displacement, stresses and strain are given below:

$$
\left.\begin{array}{l}
v=v_{0} e^{-} \frac{\mu t}{2 \eta}+y_{2} \frac{\tau_{\infty}(0)}{\mu}\left[\left(1+K t-\frac{1}{2} e^{-} \frac{\mu t}{2 \eta}-\frac{K \eta}{\mu}\left(1-e^{-} \frac{\mu t}{2 \eta}\right)\right) \cos \theta-\frac{1}{2} e^{-} \frac{\mu t}{2 \eta}\right] \\
w=w_{0} e^{-} \frac{\mu t}{2 \eta}+y_{3} \frac{\tau_{\infty}(0)}{\mu}\left[\left(1+K t-\frac{1}{2} e^{-} \frac{\mu t}{2 \eta}-\frac{K \eta}{\mu}\left(1-e^{-} \frac{\mu t}{2 \eta}\right)\right) \sin \theta-\frac{1}{2} e^{-} \frac{\mu t}{2 \eta}\right] \\
\tau_{22}=\left(\tau_{22}\right)_{0} e^{-} \frac{\mu t}{\eta}+\tau_{\infty}(0)\left[(1+K t) \cos \theta-e^{-} \frac{\mu t}{\eta}\right] \\
\tau_{23}=\left(\tau_{23}\right)_{0} e^{-} \frac{\mu t}{\eta} \\
\tau_{33}=\left(\tau_{33}\right)_{0} e^{-} \frac{\mu t}{\eta}+\tau_{\infty}(0)\left[(1+K t) \sin \theta-e^{-} \frac{\mu t}{\eta}\right] \\
E_{22}=\left(E_{22}\right)_{0} e^{-\frac{\mu t}{2 \eta}}+\frac{\tau_{\infty}(0)}{\mu}\left[\left(1+K t-\frac{1}{2} e^{-} \frac{\mu t}{2 \eta}\right.\right. \\
\left.\left.-\frac{K \eta}{\mu}\left(1-e^{-} \frac{\mu t}{2 \eta}\right)\right) \cos \theta-\frac{1}{2} e^{-} \frac{\mu t}{2 \eta}\right] \\
E_{23}=\left(E_{23}\right)^{-} \frac{\mu t}{2 \eta} \\
E_{33}=\left(E_{33}\right)_{0} e^{-\frac{\mu t}{2 \eta}}+\frac{\tau_{\infty}(0)}{\mu}\left[\left(1+K t-\frac{1}{2} e^{-\frac{\mu t}{2 \eta}}-\frac{K \eta}{\mu}\left(1-e^{-} \frac{\mu t}{2 \eta}\right)\right) \sin \theta-\frac{1}{2} e^{-} \frac{\mu t}{2 \eta}\right]
\end{array}\right\}
$$

The above solution shows that $\tau_{22}$ increases with time and $\tau_{22} \rightarrow \tau_{\infty}(t) \cos \theta$ as $t \rightarrow \infty$ while $\tau_{23} \rightarrow 0$ but $\tau_{33} \rightarrow \tau_{\infty}(t) \sin \theta$, where $\tau_{\infty}(t)=\tau_{\infty}(0)(1+K t)$.

We assume that the geological conditions as well as the characteristic of the fault is such that when the stress component $\tau_{23}$ across the fault reaches some 
critical value $\tau_{c}, \tau_{c}<\tau_{\infty}(t) \cos \theta$, the fault $F$ starts slip. For bounded stresses and strains the dislocation function $\mathrm{f}\left(\xi_{3}^{\prime}\right)$ say, should satisfy the conditions as discussed in [10]: (i) its value will be maximum on the free surface. (ii) the magnitude of the dislocation will decrease with $y_{3}^{\prime}$ as we move downwards and ultimately tends to zero near the lower edge of the fault $y_{2}^{\prime}=0, y_{3}^{\prime}=D$.

\subsection{Solution after any Fault Movement}

We assume that after time $T=75$ years the stress component $\tau_{23}$, which is the main driving force for the dip-slip motion of the fault, exceeds the critical value $\tau_{c}=250$ bar and the fault starts to slip. An additional condition characterizing the dislocation of $w$ due to the sudden movement is written in equation (8):

$$
[w]_{F}=U f\left(y_{3}^{\prime}\right) H\left(t_{1}\right), \quad[w]_{F}=\lim _{y_{2}^{\prime} \rightarrow 0^{+}} w-\lim _{y_{2}^{\prime} \rightarrow 0^{-}} w,
$$

where $\left(y_{2}^{\prime}=0,0 \leq y_{3}^{\prime} \leq D\right)$. Here $H\left(t_{1}\right)$ is the Heaviside function, $U$ is the slip magnitude and $[w]_{F}$ is the discontinuity of $w$ across $F$. Taking Laplace Transform of (8) w.r.t. time $t$, we get $\overline{[w]_{F}}=\frac{U}{p} f\left(y_{3}^{\prime}\right)$. The fault slips across $F$ after time $T=75$ years. It is to be noted that $[w]_{F}=0$ for $t_{1} \leq 0$, where $t_{1}=t-T$. The fault $F$ is located in the region $\left(y_{2}^{\prime}=0,0 \leq y_{3}^{\prime} \leq D\right)$. We note that $v$ is continuous even after the fault slip so that $v=0$, while $w$ satisfies the dislocation condition given in equation (8). The modified boundary value problem is stated below:

$$
\nabla^{2} \bar{W}=0, \text { where } \bar{W}=\bar{w}-\frac{(w)_{0}}{p+\frac{\mu}{2 \eta}},
$$

where $\bar{w}$ is the L.T. of $w$ with modified boundary conditions

$$
\left.\begin{array}{l}
\overline{\tau_{22}}\left(y_{2}, y_{3}, p\right) \rightarrow 0 \quad \text { as } \quad\left|y_{2}\right| \rightarrow \infty, y_{3} \geq 0 \\
\overline{\tau_{23}}\left(y_{2}, y_{3}, p\right) \rightarrow 0 \text { as } y_{3} \rightarrow \infty,\left(-\infty<y_{2}<\infty\right) \\
\overline{\tau_{33}}\left(y_{2}, y_{3}, p\right) \rightarrow 0 \text { as } y_{3} \rightarrow \infty,\left(-\infty<y_{2}<\infty\right)
\end{array}\right\},
$$

and all other boundary conditions are same as stated in the absence of any fault movement. We solve the above boundary value problem as shown in Appendix. Then the solution for displacements, stresses and strains after fault movement are written in following equation:

$$
\left.\begin{array}{l}
w=\frac{U}{2 \pi} \phi\left(y_{2}, y_{3}\right) H\left(t_{1}\right), \tau_{23}=\frac{U}{2 \pi} \mu\left(1+e^{-} \frac{\mu t_{1}}{\eta}\right) H\left(t_{1}\right) \phi_{1}\left(y_{2}, y_{3}\right) \\
\tau_{22}=0, \tau_{33}=\frac{U}{2 \pi} \mu\left(1+e^{-} \frac{\mu t_{1}}{\eta}\right) H\left(t_{1}\right) \phi_{2}\left(y_{2}, y_{3}\right) \\
E_{22}=0, E_{23}=\frac{U}{4 \pi} H\left(t_{1}\right) \phi_{1}\left(y_{2}, y_{3}\right), E_{33}=\frac{U}{2 \pi} H\left(t_{1}\right) \phi_{2}\left(y_{2}, y_{3}\right) .
\end{array}\right\}
$$


$H\left(t_{1}\right)$ is the Heaviside step function which gives the displacement at any point $Q\left(y_{2}, y_{3}\right)$ and $\phi\left(y_{2}, y_{3}\right), \phi_{1}\left(y_{2}, y_{3}\right), \phi_{2}\left(y_{2}, y_{3}\right)$ are given in Appendix. We try to find the solutions in the following form:

$v=(v)_{\text {absence of fault movement }}+(v)_{\text {after fault movement }}$
$w=(w)_{\text {absence of fault movement }}+(w)_{\text {after }}$ fault movement
$\tau_{22}=\left(\tau_{22}\right)_{\text {absence }}$ of fault movement $+\left(\tau_{22}\right)_{\text {after fault movement }}$
$\tau_{23}=\left(\tau_{23}\right)_{\text {absence }}$ of fault movement $+\left(\tau_{23}\right)_{\text {after }}$ fault movement
$\tau_{33}=\left(\tau_{33}\right)_{\text {absence }}$ of fault movement $+\left(\tau_{33}\right)_{\text {after }}$ fault movement
$E_{22}=\left(E_{22}\right)_{\text {absence }}$ of fault movement $+\left(E_{22}\right)_{\text {after }}$ fault movement
$E_{23}=\left(E_{23}\right)_{\text {absence }}$ of fault movement $+\left(E_{23}\right)_{\text {after }}$ fault movement
$E_{33}=\left(E_{33}\right)_{\text {absence }}$ of fault movement $+\left(E_{33}\right)_{\text {after fault movement }}$. So the final solutions are given by the following equation:

$$
\begin{aligned}
& v=v_{0} e^{-\frac{\mu t}{2 \eta}}+y_{2} \frac{\tau_{\infty}(0)}{\mu}\left[\left(1+K t-\frac{1}{2} e^{-\frac{\mu t}{2 \eta}}-\frac{K \eta}{\mu}\left(1-e^{-\frac{\mu t}{2 \eta}}\right)\right) \cos \theta-\frac{1}{2} e^{-\frac{\mu t}{2 \eta}}\right] \\
& w=w_{0} e^{-\frac{\mu t}{2 \eta}}+y_{3} \frac{\tau_{\infty}(0)}{\mu}\left[\left(1+K t-\frac{1}{2} e^{-\frac{\mu t}{2 \eta}}-\frac{K \eta}{\mu}\left(1-e^{-\frac{\mu t}{2 \eta}}\right)\right) \sin \theta-\frac{1}{2} e^{-\frac{\mu t}{2 \eta}}\right] \\
& +\frac{U}{2 \pi} \phi\left(y_{2}, y_{3}\right) H\left(t_{1}\right) \\
& \tau_{22}=\left(\tau_{22}\right)_{0} e^{-\frac{\mu t}{\eta}}+\tau_{\infty}(0)\left[(1+K t) \cos \theta-e^{-\frac{\mu t}{\eta}}\right] \\
& \tau_{23}=\left(\tau_{23}\right)_{0} e^{-\frac{\mu t}{\eta}}+\frac{U}{2 \pi} \mu\left(1+e^{-\frac{\mu t_{1}}{\eta}}\right) H\left(t_{1}\right) \phi_{1}\left(y_{2}, y_{3}\right) \\
& \left.\tau_{33}=\left(\tau_{33}\right)_{0} e^{-\frac{\mu t}{\eta}}+\tau_{\infty}(0)(1+K t) \sin \theta-e^{-\frac{\mu t}{\eta}}\right] \\
& +\frac{U}{2 \pi} \mu\left(1+e^{-\frac{\mu t_{1}}{\eta}}\right) H\left(t_{1}\right) \phi_{2}\left(y_{2}, y_{3}\right) \\
& E_{22}=\left(E_{22}\right)_{0} e^{-\frac{\mu t}{2 \eta}} \\
& +\frac{\tau_{\infty}(0)}{\mu}\left[\left(1+K t-\frac{1}{2} e^{-\frac{\mu t}{2 \eta}}-\frac{K \eta}{\mu}\left(1-e^{-\frac{\mu t}{2 \eta}}\right)\right) \cos \theta-\frac{1}{2} e^{-\frac{\mu t}{2 \eta}}\right] \\
& \left.E_{23}=\left(E_{23}\right)\right) e^{-\frac{\mu t}{2 \eta}}+\frac{U}{4 \pi} H\left(t_{1}\right) \phi_{1}\left(y_{2}, y_{3}\right)
\end{aligned}
$$

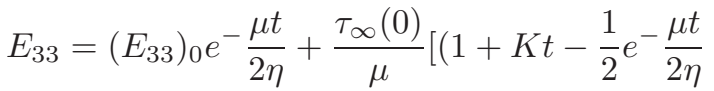

$$
\begin{aligned}
& \left.\left.-\frac{K \eta}{\mu}\left(1-e^{-\frac{\mu t}{2 \eta}}\right)\right) \sin \theta-\frac{1}{2} e^{-\frac{\mu t}{2 \eta}}\right]+\frac{U}{2 \pi} H\left(t_{1}\right) \phi_{2}\left(y_{2}, y_{3}\right) .
\end{aligned}
$$




\section{Numerical Computation}

Following Cathles [4], Aki and Richards [1] and the recent studies on rheological behaviour of the crust and upper mantle by Chift et al. [3], the value of the model parameters are taken as follows:

$\mu=3 \times 10^{10} \mathrm{~N} / \mathrm{m}^{2}$ (Pascals), $\eta=3 \times 10^{19} \mathrm{~Pa}: s$, $\tau_{\infty}(0)=30 \times 10^{5} \mathrm{~N} / \mathrm{m}^{2}, \tau_{\infty}(t)=\tau_{\infty}(0)(1+K t), \quad K=10^{-} 9$, $\mathrm{D}=$ Width of the fault $\mathrm{F}=10 \mathrm{~km}=10 \times 10^{3}$ meter,

$\left(\tau_{22}\right)_{0}=30 \times 10^{5} \mathrm{~N} / \mathrm{m}^{2}$ (Pascals), $\left(\tau_{23}\right)_{0}=30 \times 10^{5} \mathrm{~N} / \mathrm{m}^{2}$ (Pascals), $\left(\tau_{33}\right)_{0}=30 \times 10^{5} \mathrm{~N} / \mathrm{m}^{2}$ (Pascals), $\tau_{c}=250$ bar $=2.5 \times 10^{7} \mathrm{~N} / \mathrm{m}^{2}$ (Pascals), $\theta=\pi / 6, \pi / 4, \pi / 3, \pi / 2$, $\mathrm{R}=2$ meter, $\mathrm{U}=1.6$ meter, 3.7 meter, 5.6 meter.

We consider different dislocation function $f\left(\zeta_{3}^{\prime}\right)$ in the following form suggested by Godara et al. [11]:

(i) Liner Slip Function (LSF): $\mathrm{f}\left(\zeta_{3}^{\prime}\right)=\mathrm{R}\left(1-\frac{\zeta_{3}^{\prime}}{D}\right)$; (ii) Parabolic Slip Function (PSF): $\mathrm{f}\left(\zeta_{3}^{\prime}\right)=\mathrm{R}\left(1-\frac{\zeta_{3}^{\prime 2}}{D^{2}}\right)$; (iii) Elliptic Slip Function $(\mathrm{ESF}): \mathrm{f}\left(\zeta_{3}^{\prime}\right)=\mathrm{R}\left(1-\frac{\zeta_{3}^{\prime 2}}{D^{2}}\right)^{\frac{1}{2}}$, which are satisfied for all the conditions for bounded stresses and strains stated in 3.1. We have computed displacements, stresses and strains taking the above values of the parameter with new time origin $t_{1}=t-T$, where $T=75$ years (say) using MATLAB.

Fig-2(a) shows the displacement component $w$ against $y_{2}$ for different slip function taking $y_{3}=5 \mathrm{~km}$ with average slip magnitude 3.7 meter and a fixed inclination $\theta=\pi / 3$ just immediately after the fault movement. From this figure it is clear that the displacement is maximum for ESF and minimum for LSF and as we move far away from the fault, the displacement tends to zero. From Fig2(b) we have found that with the value of $y_{2}=5 \mathrm{~km}$, slip magnitude $U=3.7$ meter for the ESF, displacement is found to be maximum on the free surface $y_{3}=0$ and it sharply decreases and then gradually $\rightarrow 0$ with the increasing value of depth. This rate of decrease of displacement is higher when the fault is inclined at an angle $\theta=\pi / 6$ and this displacement falls off rapidly with the increase of inclination of the fault and finally tend to zero as $y_{3} \rightarrow \infty$ for all $\theta$.

It is observed that the slip is small for earthquake of smaller magnitude and this slip is higher for earthquake of higher magnitude.

Fig-3(a) shows the variation of displacement $w$ with $y_{2}$ for different slip magnitude. It is found that for $y_{3}=5 \mathrm{~km}$ with fixed dip angle and ESF, the displacement is maximum for maximum slip magnitude (5.6 meters) and it is symmetric about $y_{2} \approx 0$. Shear stress $\tau_{23}$ with $y_{2}$ has been shown in Fig-3(b) for various inclinations taking $y_{3}=0$ with average slip magnitude and ESF. This 


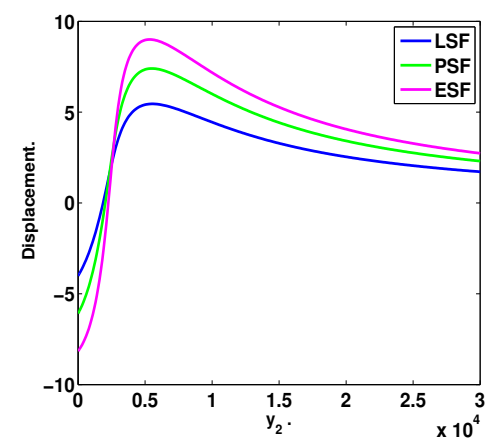

(a)

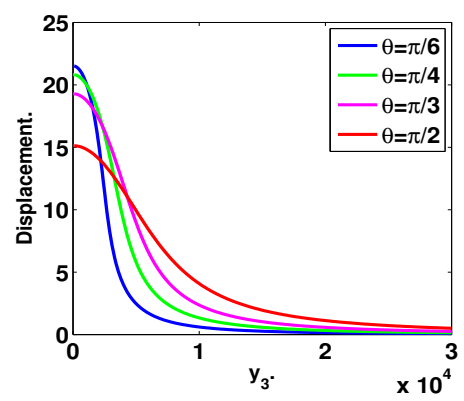

(b)

Figure 2: (a): Displacement $w$ against $y_{2}$ for different slip function. (b): Displacement $w$ against $y_{3}$ for various inclination.

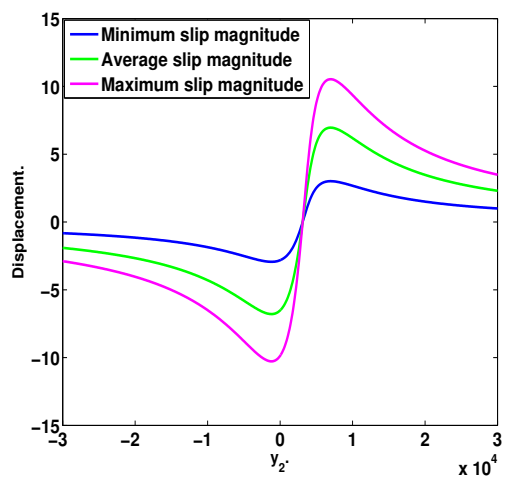

(a)

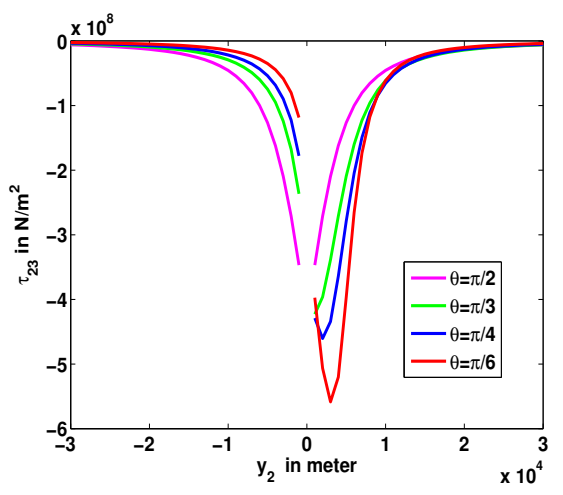

(b)

Figure 3: (a): Displacement $\mathrm{w}$ with $y_{2}$ for different slip magnitude. (b): Stress $\tau_{23}$ with $y_{2}$ for various dip angle.

shows that when $\left|y_{2}\right| \rightarrow \infty$ this stress approaches to zero and finally vanishes. Each stress curve has a branch cut which reflects the discontinuity in stress near the fault.

For fixed dip angle $\theta=\pi / 3$ and $y_{2}=5 \mathrm{~km}$, shear stress $\tau_{23}$ is plotted against $y_{3}$ for different slip function in Fig-4(a). The accumulation of stress $\tau_{23}$ is maximum near $y_{3}=5 \mathrm{~km}$ and this maximum value is attained quite rapidly for ESF and comparatively slow for LSF. All the stresses are found to tend to zero as $y_{3} \rightarrow \infty$ from free surface. 


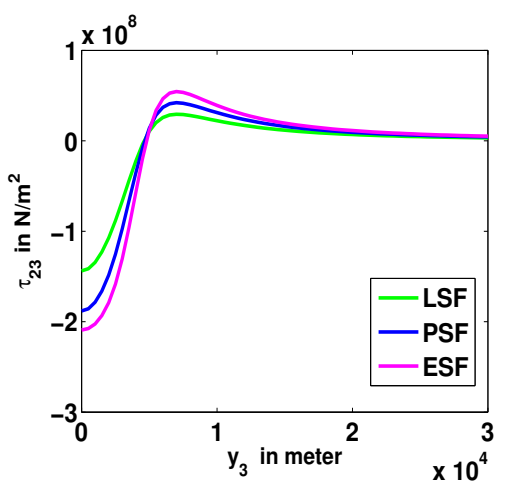

(a)

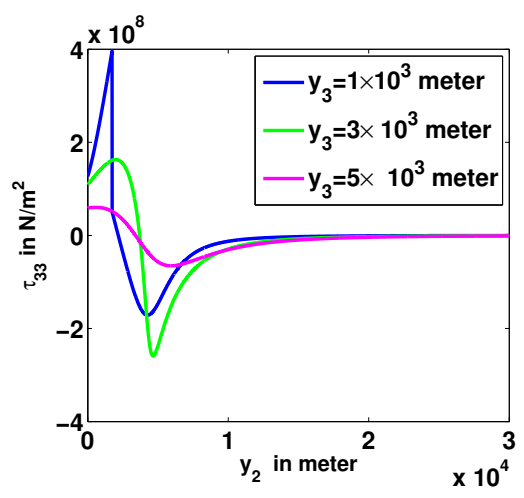

(b)

Figure 4: (a): Stress $\tau_{23}$ with $y_{3}$ for various slip function. (b): Stress $\tau_{33}$ with $y_{2}$ for various $y_{3}$.

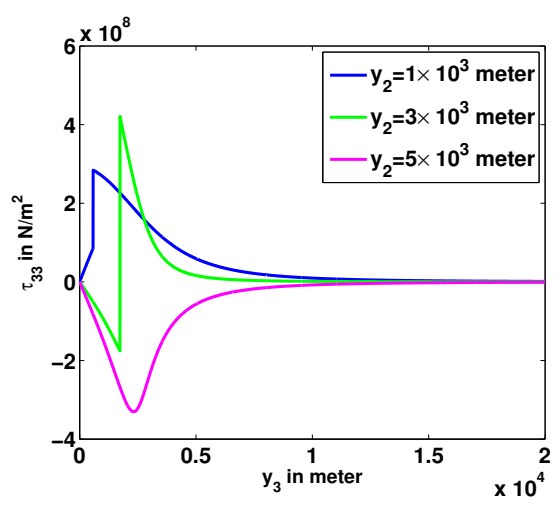

(a)

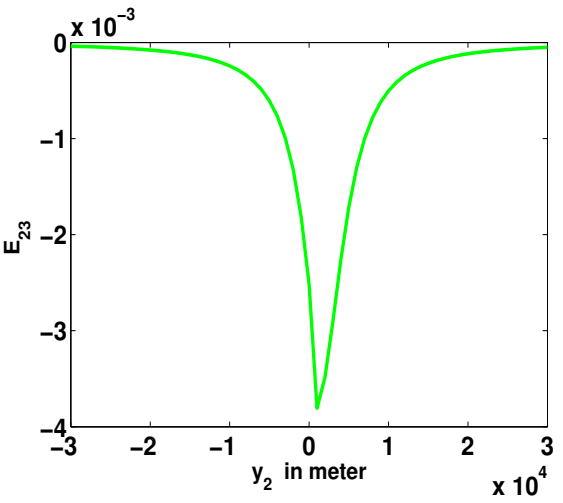

(b)

Figure 5: (a): Stress $\tau_{33}$ with $y_{3}$ for various $y_{2}$. (b): Strain $E_{23}$ with $y_{2}$.

Fig-4(b) shows normal stress for a fixed inclination with $y_{2}$ for various $y_{3}$, taking parabolic slip function and average slip magnitude. We have found that for $y_{3}=1 \mathrm{~km}$, the normal stress $\tau_{33}$ increases sharply up to certain value after that it decreases rapidly and $\rightarrow 0$ as expected. For $y_{3}=3 \mathrm{~km}$, there is gradual increase of stress and then it decreases. For $y_{3}=1 \mathrm{~km}$, the rate of decrease of stress is higher than the other values of $y_{3}$.

In Fig-5(a), this normal stress is plotted against $y_{3}$ for different $y_{2}$. Here normal stress suddenly increases near the fault for $y_{2}=1 \mathrm{~km}$, but maximum stress 


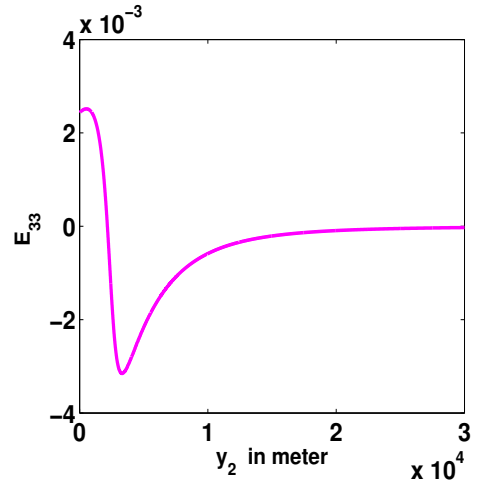

(a)

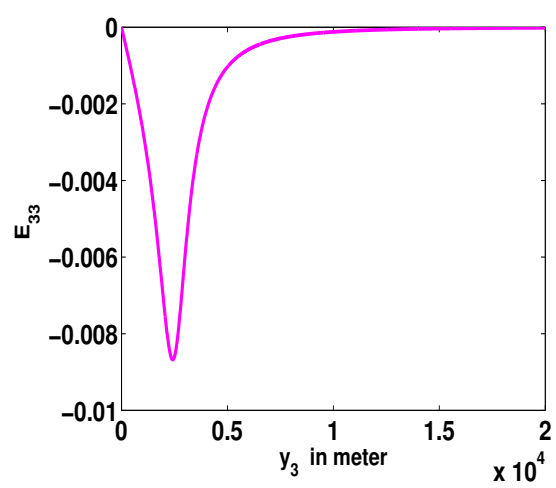

(b)

Figure 6: (a): Strain $E_{33}$ with $y_{2}$. (b): Strain $E_{33}$ with $y_{3}$.

occurs when $y_{2}=3 \mathrm{~km}$, little bit away from the fault, it is about $4.2 \times 10^{8} \mathrm{~N} / \mathrm{m}^{2}$. For $y_{2}=5 \mathrm{~km}$, stress is negative everywhere and finally it tends to zero. Fig5 (b) shows the shear strain $\left(E_{23}\right)$ release with $y_{2}$, taking $y_{3}=1 \mathrm{~km}$, and $\theta=\pi / 3$ with average slip magnitude and elliptic type slip function. It shows that the release of shear strain near the free surface is maximum near the fault $y_{2}=0$ and this effect gradually falls off as we move far away from the trace of the fault. This shear strain release is negative everywhere because after fault movement stress is releasing so corresponding strain also releases and hence it is negative everywhere. We have also observed that for $y_{3}=0$ this strain has a branch cut about $y_{2}=0$ as expected.

Fig-6(a) shows the normal strain $E_{33}$ with $y_{2}$ taking $y_{3}=5 \mathrm{~km}, \theta=\pi / 3$, slip magnitude of 1.5 meter but slip function of parabolic type. There is a strain accumulation near the fault $y_{2} \approx 0$. This accumulation decreases sharply for $0<y_{2}<0.5 \mathrm{~km}$, i.e. there is release of strain for $y_{2}>0$ and it approaches to zero for $y_{2} \rightarrow \infty$. In Fig- 6(b), same normal strain has been plotted against $y_{3}$ taking $y_{2}=5 \mathrm{~km}, \theta=\pi / 6$ and $U=3.7$ meter but slip function is elliptic type. The pattern of strain is almost same as in Fig-6(a) but for present case strain release is more sharper and it tends to zero very rapidly within shallow depth.

\section{Conclusions}

The contribution of the previous study [8] suggest that the Rheological properties of standard linear solid (SLS) may be a suitable representation of the 
lithosphere-asthenosphere system. So we derived analytical solutions for displacement, stress and strain due to fault movement across an inclined, infinite, Dip-slip fault situated in a visco-elastic half space of Maxwell and Kelvin-Voigt type material. From the above numerical computation, it is found that these displacement, stresses and strains depend on various inclinations and slip magnitude of the fault. Also the stress accumulation / release near the fault varies not only due to dip angle and slip magnitude but also on slip functions and visco-elastic material in the lithosphere-asthenosphere system. The values of the model parameters play an important role in determining the displacement, stress and strain. The movement of fault causes stress accumulation/release near the fault which essentially depend on different positions of point on the fault for fixed width.

\section{Appendix}

Taking the Laplace transform of all constitutive equations and boundary conditions, one can obtain

$$
\overline{\tau_{22}}=\frac{\eta\left(\tau_{22}\right)_{0}}{\mu\left(1+\frac{\eta p}{\mu}\right)}+\frac{(\mu+2 \eta p) \frac{\partial \bar{v}}{\partial y_{2}}}{1+\frac{\eta p}{\mu}}-\frac{2 \eta\left(\frac{\partial v}{\partial y_{2}}\right)_{0}}{1+\frac{\eta p}{\mu}}
$$

and similar other equation for $\overline{\tau_{23}}$ and $\overline{\tau_{33}}$.

Also we have the boundary condition in transform domain as:

$$
\left.\begin{array}{rl}
\overline{\tau_{22}}\left(y_{2}, y_{3}, t\right) & =\tau_{\infty}(0)\left(\frac{1}{p}+\frac{K}{p^{2}}\right) \cos \theta \text { as }\left|y_{2}\right| \rightarrow \infty \\
\overline{\tau_{23}}\left(y_{2}, y_{3}, t\right) & =0 \text { as } y_{3} \rightarrow \infty, \\
\overline{\tau_{33}}\left(y_{2}, y_{3}, t\right) & =\tau_{\infty}(0)\left(\frac{1}{p}+\frac{K}{p^{2}}\right) \sin \theta \text { as }\left|y_{2}\right| \rightarrow \infty, \\
\left(-\infty<y_{2}\right. & <\infty, \quad t \geq 0),\left(y_{3} \geq 0, \quad t \geq 0\right), \quad K>0
\end{array}\right\}
$$

Here $\tau_{\infty}(0)$ is the value of $\tau_{\infty}(t)$ at $t=0$, where $\overline{\tau_{22}}=\int_{0}^{\infty} \tau_{22} e^{-p t} d t, p$ being Laplace transform variable.

We have equation (6), in transform domain it can be written as,

$\nabla^{2} \bar{V}=0$, where $\bar{V}=\bar{v}-\frac{v_{0}}{p+\frac{\mu}{2 \eta}}, \quad \nabla^{2} \bar{W}=0$, where $\bar{W}=\bar{w}-\frac{w_{0}}{p+\frac{\mu}{2 \eta}}$.

We solve the governing Laplace equation with the boundary conditions (3)(5) and (10). 
To solve the boundary value problem, one might guess that $\bar{v}, \bar{w}$ have the form $\bar{v}\left(y_{2}, y_{3}\right)=\frac{v_{0}}{p+\frac{\mu}{2 \eta}}+A y_{2}+B y_{3}$ and $\bar{w}\left(y_{2}, y_{3}\right)=\frac{w_{0}}{p+\frac{\mu}{2 \eta}}+C y_{2}+D y_{3}$. Using the initial and boundary conditions and taking inverse Laplace transform, the solution of displacement, stress and strain before any fault movement is given by the equation (7). After the fault movement, an additional uniform dislocation condition which characterizes the sudden movement across $F$ is given by equation (8). Taking L.T. of (8), we get

$$
\overline{[w]}=\frac{U}{s} f\left(y_{3}^{\prime}\right) .
$$

All the basic equations and initial conditions are same as before for the post movement period. The modified boundary conditions are given in equation (10). We solved the above boundary value problem by modified Green's function method developed by Maruyama [12], [13] and Rybicki [16], [17] and correspondence principle. Let $Q\left(y_{2}, y_{3}\right)$ be any point in the medium and $P\left(\zeta_{2}, \zeta_{3}\right)$ be any point on the fault $F$, then we have

$$
\bar{w}(Q)=\int_{F} \bar{w}(P) G(P, Q),
$$

where $G(P, Q)=G_{32}^{3}(P, Q) d \zeta_{3}-G_{33}^{3}(P, Q) d \zeta_{2}$

and $G_{32}^{3}(P, Q), G_{33}^{3}(P, Q)$ are

$G_{32}^{3}(P, Q)=\frac{1}{2 \pi}\left[\frac{y_{2}-\zeta_{2}}{L^{2}}+\frac{y_{2}-\zeta_{2}}{M^{2}}\right], G_{33}^{3}(P, Q)=\frac{1}{2 \pi}\left[\frac{y_{3}-\zeta_{3}}{L^{2}}-\frac{y_{3}+\zeta_{3}}{M^{2}}\right]$,

$L^{2}=\left(y_{2}-\zeta_{2}\right)^{2}+\left(y_{3}-\zeta_{3}\right)^{2}, \quad M^{2}=\left(y_{2}-\zeta_{2}\right)^{2}+\left(y_{3}+\zeta_{3}\right)^{2}$.

Now $\left(\zeta_{2}, \zeta_{3}\right)$ being a point on $F, 0 \leq \zeta_{2} \leq D \cos \theta, 0 \leq \zeta_{3} \leq D \sin \theta$ and $\zeta_{2}=$ $\zeta_{3} \cot \theta$. A change in the co-ordinate axis from $\left(\zeta_{2}, \zeta_{3}\right)$ to $\left(\zeta_{2}^{\prime}, \zeta_{3}^{\prime}\right)$ is connected by the relation: $\zeta_{2}=\zeta_{2}^{\prime} \sin \theta+\zeta_{3}^{\prime} \cos \theta, \zeta_{3}=-\zeta_{2}^{\prime} \cos \theta+\zeta_{3}^{\prime} \sin \theta$ so that $\zeta_{2}^{\prime}=$ $0,0 \leq \zeta_{3}^{\prime} \leq D$ on $F$. Therefore $d \zeta_{2}=\cos \theta d \zeta_{3}^{\prime}, d \zeta_{3}=\sin \theta d \zeta_{3}^{\prime}$. Thus,

$$
\bar{w}(Q)=\frac{U}{2 \pi p} \int_{0}^{D}\left[\frac{y_{2} \sin \theta-y_{3} \cos \theta}{L^{2}}+\frac{y_{2} \sin \theta+y_{3} \cos \theta}{M^{2}}\right] f\left(\zeta_{3}^{\prime}\right) d \zeta_{3}^{\prime} .
$$

Taking the inverse Laplace transform, we get

$$
w(Q)=\frac{U}{2 \pi} \phi\left(y_{2}, y_{3}\right) H\left(t_{1}\right)
$$

where $\phi\left(y_{2}, y_{3}\right)=\int_{0}^{D}\left[\frac{y_{2} \sin \theta-y_{3} \cos \theta}{L^{2}}+\frac{y_{2} \sin \theta+y_{3} \cos \theta}{M^{2}}\right] f\left(\zeta_{3}^{\prime}\right) d \zeta_{3}^{\prime}$ and $L^{2}, M^{2}$ have the form $L^{2}=\zeta_{3}^{\prime 2}-2 \zeta_{3}^{\prime}\left(y_{2} \cos \theta+y_{3} \sin \theta\right)+y_{2}^{2}+y_{3}^{2}$, $M^{2}=\zeta_{3}^{\prime 2}-2 \zeta_{3}^{\prime}\left(y_{2} \cos \theta-y_{3} \sin \theta\right)+y_{2}^{2}+y_{3}^{2}$.

It is to be noted that $w=0$ for $t_{1}=t-T \leq 0$. From the equation (13), (17) and assuming displacement, stress and strain to be zero for $t_{1}=t-T<0$, 
we have $\bar{\tau}_{22}=0$. We note that $v$ is continuous even after the fault slip so that $v=0$ after fault movement. Taking inverse Laplace transform, $\tau_{22}=0$. Similarly other equations are as follows:

$$
\begin{aligned}
& \tau_{23}=\frac{U}{2 \pi} \mu H\left(t_{1}\right)\left(1+e^{-\frac{\mu t_{1}}{\eta}}\right) \phi_{1}\left(y_{2}, y_{3}\right) \\
& E_{23}=\frac{U}{4 \pi} \mu\left(1+e^{-\frac{\mu t_{1}}{\eta}}\right) H\left(t_{1}\right) \phi_{1}\left(y_{2}, y_{3}\right), \text { where } \phi_{1}\left(y_{2}, y_{3}\right)=\frac{\partial \phi}{\partial y_{2}} \\
& =\int_{0}^{D}\left[\frac{\zeta_{3}^{\prime 2} \sin \theta-2 y_{3}\left(\zeta_{3}^{\prime}-y_{2} \cos \theta\right)-\left(y_{2}^{2}-y_{3}^{2}\right) \sin \theta}{L^{4}}\right. \\
& \begin{array}{l}
\left.+\frac{\zeta_{3}^{\prime 2} \sin \theta+2 y_{3}\left(\zeta_{3}^{\prime}-y_{2} \cos \theta\right)-\left(y_{2}^{2}-y_{3}^{2}\right) \sin \theta}{M^{4}}\right] f \\
\tau_{33}=\frac{U}{2 \pi} \mu H\left(t_{1}\right)\left(1+e^{-\frac{\mu t_{1}}{\eta}}\right) \phi_{2}\left(y_{2}, y_{3}\right), \quad E_{22}=0
\end{array} \\
& E_{33}=\frac{U}{2 \pi} \mu\left(1+e^{-\frac{\mu t_{1}}{\eta}}\right) H\left(t_{1}\right) \phi_{2}\left(y_{2}, y_{3}\right), \text { where } \phi_{2}\left(y_{2}, y_{3}\right)=\frac{\partial \phi}{\partial y_{3}} \\
& =-\int_{0}^{D}\left[\frac{\zeta_{3}^{\prime 2} \cos \theta-2 y_{2}\left(\zeta_{3}^{\prime}-y_{3} \sin \theta\right)+\left(y_{2}^{2}-y_{3}^{2}\right) \cos \theta}{L^{4}}\right. \\
& \left.-\frac{\zeta_{3}^{\prime 2} \cos \theta-2 y_{2}\left(\zeta_{3}^{\prime}+y_{3} \sin \theta\right)+\left(y_{2}^{2}-y_{3}^{2}\right) \cos \theta}{M^{4}}\right] f\left(\zeta_{3}^{\prime}\right) d \zeta_{3}^{\prime}
\end{aligned}
$$

\section{Acknowledgements}

The author, Debabrata Mondal acknowledges the financial assistance received from RGNF, a research scheme under U.G.C., Government of India.

\section{References}

[1] K. Aki, P.G. Richards, Quantitative Seismology: Theory and Methods, W.H. Freeman, San Francisco (1980).

[2] D.J. Andrews, Evaluation of static stress on a fault plain from a Green's function, Bull. Seis. Soc. Am., 64 (1974), 1629-1633.

[3] P. Chift, J. Lin, and U. Barckhausen, Evidence of low flexural rigidity and low viscosity lower continental crust during continental break-up in South China Sea, Marine and Petroleum Geology, 19 (2002), 951-970. 
[4] L.M. Cathles, The Viscosity of the Earth's Mantle, Princeton University Press, Princeton, New Jersey (1975).

[5] M.A. Chinnery, The deformation of the ground around surface faults, Bull. Seis. Soc. Am., 51 (1961), 355-372.

[6] S.C. Cohen, Post seismic viscoelastic surface deformations and stress 1. Theoretical considerations, displacements, and strains calculations, J. Geophys. Res., 85, B6 (1980), 3131-3150.

[7] S.K. Debnath, A buried vertical Long dip-slip fault in a Viscoelastic halfspace model of the lithosphere, J. of Emerging Trends in Engineering and Appl. Sci. (JETEAS), 4, No 1 (2013), 7-15.

[8] P. Debnath, S. Sen, Two neighbouring strike slip faults and their interaction, IOSR J. of Appl. Geology and Geophysics, 2, No 6.ver.-1 (2014), 44-56.

[9] L.B. Freund, D.M. Barnett, A two-dimensional analysis of surface deformation due to dip-slip faulting, Bull. Seis. Soc. Am., 66, No 3 (1976), 667-675.

[10] U. Ghosh, A. Mukhopadhyay and S. Sen, On two interacting creeping vertical surface-breaking strike-slip faults in two-layered model of lithosphere, Physics of the Earth and Planetary Interiors, 70 (1992), 119-129.

[11] Y. Godara, R.K. Sahrawat and M. Sing, Static elastic deformation in an orthotropic half space with rigid boundary model due to non uniform long strike slip fault, J. Earth Syst. Sci., 126, No 97 (2017), 1-10.

[12] T. Maruyama, Statical elastic dislocations in an infinite and semi-infinite medium, Bull. Earthquake Res. Inst., 42 (1964), 289-368.

[13] T. Maruyama, On two dimensional elastic dislocations in an infinite and semi-infinite medium, Bull. Earthquake Res. Inst., 44, (1966), 811-871.

[14] A. Mukhopadhyay, On stress accumulation and fault slip in lithosphere, Indian J. of Meteorology, Hydrology and Geophysics (Mausam), 30 (1979), 353-358.

[15] A. Mukhopadhyay, S. Sen and B.P. Paul, On stress accumulation in a viscoelastic lithosphere containing a continuously slipping fault, Bull. Soc. Earthquake Techonology, 17, No 1 (1980), 1-10. 
[16] K. Rybicki, Dislocation and their geophysical applications; in: Continuum Theories in Solid Earth Physics, (ed. R. Tiesseyre), Elsevier, Amsterdam (1986), 18-186.

[17] K. Rybicki, The elastic residual field of a very long strike slip fault in the presence of a discontinuity, Bull. Seis. Soc. Am., 61, No 1 (1971), 79-92.

[18] J.C. Savage and L.M. Hastie, Surface Deformation associated with dip-slip faulting, J. Geophys. Res., 71 (1966), 4897-4907.

[19] P. Segall, Earthquake and Volcano Deformation, Princeton Univ. Press (2010).

[20] S.J. Sing and S. Rani, 2-D modelling of crustal deformation associated with strike-slip and dip-slip faulting in the earth, Proc. Nat. Acad. Sci. India, LXVI (1996), 187-215.

[21] S. Sen and S.K. Debnath, Long dip-slip Fault in a viscoelastic half space model of the lithosphere, American J. of Comput. and Appl. Math., 2, No 6 (2012), 249-256.

[22] J.A. Steketee, On Volterra's dislocations in a semi-infinite elastic medium, Canad. J. Phys., 36 (1958), 192-205.

[23] J.A. Steketee, Some geophysical applications of the elasticity theory of dislocations, Can. J. Phys., 36 (1958), 1168-1198.

[24] S.K. Tomar and N.K. Dhiman, 2-D deformation analysis of a half-space due to a long dip-slip fault at finite depth, Proc. Indian Acad. Sci. (Earth Planet. Sci.), 112, No 4 (2003), 587-596. 